\title{
El incremento de la hidratación parenteral durante el trabajo de parto en mujeres nulíparas disminuye la frecuencia de parto prolongado
}

A Randomized controlled trial of the effect of increased intravenous hydration on the course of labor in nulliparous women. Garite T.,Weeks J, Peters-Pahair K.. Am J Obstet Gynecol 2000;183:1544-8.

\section{Objetivo}

Evaluar si el incremento de la hidratación parenteral durante el trabajo de parto en mujeres nulíparas afecta la progresión del trabajo de parto.

\section{Diseño}

Ensayo clínico aleatorizado.

Lugar

Universidad de California, EE.UU.

\section{Pacientes}

Mujeres nulíparas en fase activa del trabajo de parto espontáneo, de término, en presentación cefálica.

\section{Intervención}

Se aleatorizó a las pacientes para recibir $125 \mathrm{ml} /$ hora $(\mathrm{n}=94)$ o 250 $\mathrm{ml} /$ hora $(\mathrm{n}=101)$ de solución fisiológica o de ringer lactato vía parenteral. Otras intervenciones (rotura artificial de las membranas ovulares, uso de narcóticos, ocitocina, analgesia peridural) se utilizaron según decisiones del médico obstetra.

\section{Medición de resultados principales}

El resultado principal lo constituyó la disminución en la duración del trabajo de parto en minutos, según curvas de duración propias de la institución, considerando como parto prolongado aquel de más de 12 horas de duración.

\section{Resultados Principales}

La frecuencia de parto prolongado fue mayor en el grupo 125 que en el de $250 \mathrm{ml} / \mathrm{h}$ ( 26 vs. $13 \%$, p 0.04). Asimismo se observó una tendencia a menor duración de la fase latente y de la duración total del trabajo de parto en el grupo $250 \mathrm{ml} / \mathrm{h}(p=0.06)$; no así en las pacientes que culminaron en operación cesarea $(p=0.22)$. Secundariamente se observó una tendencia a mayor utilización de ocitocina en el grupo $125 \mathrm{ml} / \mathrm{h} \quad(p=0.062)$.

\section{Conclusiones}

El incremento de la hidratación parenteral durante el trabajo de parto en mujeres nulíparas se asoció a una disminución en la ocurrencia de parto prolongado.

\section{Comentario}

Los factores que intervienen en la progresión del trabajo de parto han sido analizados de manera exhaustiva, con el objetivo de comprender su fisiología así como para tratar sus anormalidades. La alimentación y la hidratación durante el trabajo de parto han sido poco estudiados, y son motivo de controversia. ${ }^{1}$

En la práctica obstétrica habitualmente se restringe la alimentación y la ingesta de líquidos vía oral intentando evitar el reflujo gastroesofágico si la embarazada debe ser sometida a una anestesia general, y se la hidrata por vía parenteral con dosis arbitrarias. A pesar de ser una intervención rutinaria, la hidratación parenteral ha sido estudiada mínimamente y aún no conocemos como afecta esta intervención la progresión del trabajo de parto.2 Existen numerosos estudios que valoran la eficacia de un adecuado reemplazo de los líquidos en maratonistas y ciclistas, los cuales demuestran una mejoría en su desempeño deportivo.3,4 A partir de estudios acerca de la fisiología muscular en el ejercicio, se especula acerca del impacto de la reposición de líquidos sobre la perfusión uterina y la dinámica del músculo uterino. Tal es así que la hidratación adecuada podría teóricamente mejorar la performance del músculo uterino.

Los autores encontraron que la hidratación a mayores volúmenes (250 ml/hora) disminuyó la probabilidad de parto prolongado.

Desde el punto de vista metodológico, deben observarse algunas críticas: un insuficiente tamaño muestral, la presencia de cointervenciones, la falta de enmascaramiento, y una presentación inadecuada de los resultados - limitación reconocida por los autores del articulo-.

Aun suponiendo que la fisiología del ejercicio sea igual en estos dos tipos de músculos diferentes (estriado y liso), se debe confirmar específicamente los resultados clínicos de estas intervenciones sobre el músculo miometrial.

En resumen, aun con ciertas reservas respecto del diseño de la investigación, este trabajo abre una perspectiva de estudio futura de una práctica habitualmente utilizada.

Dr. Alejandro M. Oubiña [ Servicio de Obstetricia. Hospital Britanico de Buenos Aires ]

Referencias

1. Macnaughton MC. Clin Obstet Gynecol 1982 Mar;25(1):137-44

2. Sommer PA, Norr K, Roberts J. Clinical decision-making regarding intravenous hydration in normal labor in a birth center setting. J Midwifery Womens Health. 2000 Mar-Apr;45(2):114- 